\title{
Stabilization of Delta Operator Systems with Actuator Saturation via an Anti-Windup Compensator
}

\author{
Hafsaa Rachid, Ouarda Lamrabet * and El Houssaine Tissir \\ LESSI, Department of Physics, Faculty of Sciences Dhar El Mehraz, University of Sidi Mohammed Ben Abdellah, \\ Fes B.P. 1796, Fes-Atlas 30000, Morocco \\ * Correspondence: ouarda.lamrabet@usmba.ac.ma
}

Received: 25 June 2019; Accepted: 16 August 2019; Published: 29 August 2019

\begin{abstract}
The design of an anti-windup controller for delta operator systems with time-varying delay and actuator saturation is addressed. By utilizing the input-output approach and three-term approximation, we first transform the original system into two equivalent interconnected subsystems. Then, by employing the scaled small-gain theorem, the Lyapunov-Krasovskii functional, and Wirtinger's integral inequality, sufficient conditions for the synthesis of an anti-windup compensator are presented in the form of linear matrix inequalities (LMIs). The estimated domain of attraction is maximized by an optimization algorithm. Numerical examples are studied to show the merits of the proposed technique.
\end{abstract}

Keywords: delta operator system; actuator saturation; anti-windup compensator; scaled small gain theorem

\section{Introduction}

Time-delay frequently arises in various practical control systems, such as communication systems, power systems, and chemical systems. The existence of delays can lead to instability and degrades the performances of systems. Thus, the time-delayed systems have been an active area of research for many years, and many effective methods have been developed, such as the delay-partitioning approach [1], the free weighting matrices technique [2,3], the Wirtinger inequality approach [4], and the generalized free-matrix-based integral inequality [5]. The input-output (IO) approach is also an appropriate approach for time-delayed systems, its main idea being to transform the original systems into two interconnection subsystems. Based on the IO approach and the scaled small gain (SSG) theorem, a new time-varying delay approximation model by employing a three-term approximation was first proposed in [6].

It is important to note that discrete systems are suitable for computer realization, and continuous systems are convenient for theoretical analysis. The unique framework that can combine some related results in both continuous and discrete domains is the delta operator approach $[7,8]$. Recently, a great deal of attention has been paid to the analysis and synthesis of delta operator systems. For example, the problem of asymptotic stability and stabilization of delta operator systems with uncertainties and time-varying delays was studied in [9]. In Reference [10], the authors used the delta operator approach to investigates the robust $H_{\infty}$ filtering design for T-Sfuzzy systems with uncertainties and time-varying delay. In Reference [11], the robust stability of delta operator systems was discussed via the three-term approximation approach.

On the other side, due to the fact that physical inputs are usually limited, actuator saturation is very ubiquitous in all practical control systems. The controller design without taking into account the saturation effects can lead to performance degradation, causes the instability of the closed-loop system, and could even involve security problems, such as crashes of the aircraft JAS Gripen and 
the meltdown of the Chernobyl power plant. Therefore, people are paying more and more attention to control systems with actuator saturation [12-14]. A common technique to avoid the effect of saturation is the anti-windup compensator. A large amount of research results about the anti-windup compensator exists in the literature. In [15], the authors addressed the problem of anti-windup design for sampled data with time delay and input saturation. The input delay approach was used to develop an anti-windup compensator for Active Queue Management (AQM) in Transmission Control Protocol/Internet Protocol (TCP/IP) networks in [16]. Some efforts were devoted to the problem of delta operator systems under input limitations; see for example [17,18]. However, there have been no result on the delta operator systems with input limitations via the anti-windup design, so this motivates the current study.

The aim of this paper is to synthesize an anti-windup compensator for delta operator systems with saturating control and time-varying delay. By incorporating the IO approach, Wirtinger's integral inequality, and the Lyapunov-Krasovskii functional, sufficient conditions are derived by means of linear matrix inequalities (LMIs), ensuring the asymptotic stability of the system for any initial condition inside an estimate of the domain of attraction. An optimization procedure is proposed to enlarge the region of initial conditions that ensure the stability of the system. Further, numerical simulation examples are presented to illustrate the significance of the proposed results.

Notation 1. We use the following notation to illustrate the letters and symbols in the relevant analysis. We let $t_{k}=k T$, where $T$ is the sampling period of a system. $\mathbf{G}_{\mathbf{1}} \circ \mathbf{G}_{\mathbf{2}}$ represents the series connection of mappings $\mathbf{G}_{\mathbf{1}}$ and $\mathbf{G}_{2} \cdot \bar{\lambda}(P)$ denotes the maximal eigenvalue of a matrix $P$. diag $\{\ldots\}$ denotes a block-diagonal matrix. $\|x\|_{l_{2}} \triangleq \sqrt[2]{\sum_{k=0}^{\infty} x^{T}(k) x(k)}$ denotes the $l_{2}$ norm of series $x(k)$, and $\|.\|_{\infty}$ represents the $l_{2}$-induced norm of a transfer function matrix or a general operator.

\section{Problem Statement and Preliminaries}

As in $[7,19]$, the delta operator is defined by:

$$
\delta x\left(t_{k}\right)= \begin{cases}\frac{d x(t)}{d(t)}, & T=0 \\ \frac{x\left(t_{k}+T\right)-x\left(t_{k}\right)}{T}, & T \neq 0\end{cases}
$$

where $\lim \left(\delta x\left(t_{k}\right)\right)=\dot{x}(t)$ when $T \longrightarrow 0^{+}, T$ is a sampling period, $t$ is the continuous time, and $k$ is the time step with $t_{k}=k T$. The delta operator system with time-varying delay is described as:

$$
\begin{aligned}
(S): \delta x\left(t_{k}\right) & =A x\left(t_{k}\right)+A_{d} x\left(t_{k}-r_{h}\right)+B u\left(t_{k}\right) \\
q\left(t_{k}\right) & =C_{q} x\left(t_{k}\right) \\
x\left(t_{k}\right) & =\varphi\left(t_{k}\right), t_{k}=-r_{h 2},-r_{h 2}+T, \ldots, 0
\end{aligned}
$$

where $x\left(t_{k}\right) \in \mathcal{R}^{n}, u\left(t_{k}\right) \in \mathcal{R}^{m}, q\left(t_{k}\right) \in \mathcal{R}^{p}$ are the state vector, control vector, and measured output, respectively, with $A, A_{d}$ and $C_{q}$ constant real matrices, $r_{h}$ is the time-varying delay that ensures $r_{h}=h T, r_{h 1} \leq r_{h} \leq r_{h 2}, 1 \leq h_{1} \leq h \leq h_{2}$, where $r_{h 1}$ and $r_{h 2}$ are known constants and $T$ is the sampling period, and $\varphi\left(t_{k}\right), t_{k} \in\left[-r_{h 2}, 0\right]$ is a real-valued initial function.

Remark 1. Note that for continuous systems $(T=0)$, the time delay is assumed to be a bounded function. However, when discretizing this function, it becomes dependent on the sampling period, and an additional assumption is added. That is, the time delay is a multiple of $T$, mathematically $r_{h}=h T$. Note that this assumption is applied only on the discrete time domain.

To control System (2), let a dynamic stabilizing controller in the following form,

$$
\begin{aligned}
\delta x_{c}\left(t_{k}\right) & =A_{c} x_{c}\left(t_{k}\right)+B_{c} q\left(t_{k}\right) \\
y_{c}\left(t_{k}\right) & =C_{c} x\left(t_{k}\right)+D_{c} q\left(t_{k}\right) .
\end{aligned}
$$


where $x_{c}\left(t_{k}\right)$ is the controller state, $u_{c}\left(t_{k}\right)=q\left(t_{k}\right)$ is the controller input, and $y_{c}\left(t_{k}\right)$ is the controller output. $A_{c}, B_{c}, C_{c}$, and $D_{c}$ are known matrices of appropriate dimensions.

Due to the existence of saturation, the control input of the system can be expressed as:

$$
u\left(t_{k}\right)=\operatorname{sat}\left(y_{c}\left(t_{k}\right)\right)
$$

where sat $\left(y_{c_{(i)}}\left(t_{k}\right)\right)=\operatorname{sign}\left(y_{c_{(i)}}\left(t_{k}\right)\right) \min \left\{\left|y_{c_{(i)}}\left(t_{k}\right)\right|, u_{0_{(i)}}\right\}, i=1, \ldots, m$

In order to overcome the undesirable effects caused by saturation, we add the anti-windup signal such that,

$$
\begin{aligned}
\delta x_{c}\left(t_{k}\right) & =A_{c} x_{c}\left(t_{k}\right)+B_{c} y\left(t_{k}\right)-E_{c} \psi\left(y_{c}\left(t_{k}\right)\right) \\
y_{c}\left(t_{k}\right) & =C_{c} x_{c}\left(t_{k}\right)+D_{c} q\left(t_{k}\right)
\end{aligned}
$$

where $\psi\left(y_{c}\left(t_{k}\right)\right)$ is a decentralized dead-zone nonlinearity, such that $\left.\psi\left(y_{c}\left(t_{k}\right)\right)=y_{c}\left(t_{k}\right)\right)-\operatorname{sat}\left(y_{c}\left(t_{k}\right)\right)$. Define $\xi(t)=\left[\begin{array}{lll}x^{T}\left(t_{k}\right) & x_{c}^{T}\left(t_{k}\right)\end{array}\right]^{T}$, then we obtain the closed-loop system as follows:

$$
\delta \xi\left(t_{k}\right)=\mathbb{A} \xi\left(t_{k}\right)+\mathbb{A}_{d} \xi\left(t_{k}-r_{h}\right)-\left(\mathbb{B}+\mathbb{R} E_{c}\right) \psi\left(\mathbb{K} \xi\left(t_{k}\right)\right)
$$

where:

$$
\begin{aligned}
& \mathbb{A}=\left[\begin{array}{cc}
A+B D_{c} C_{q} & B C_{c} \\
B_{c} C_{q} & A_{c}
\end{array}\right], \mathbb{B}=\left[\begin{array}{l}
B \\
0
\end{array}\right], \mathbb{A}_{d}=\left[\begin{array}{cc}
A_{d} & 0 \\
0 & 0
\end{array}\right] \\
& \mathbb{R}=\left[\begin{array}{c}
0 \\
I_{n_{c}}
\end{array}\right], \mathbb{K}=\left[\begin{array}{ll}
D_{c} C_{q} & C_{c}
\end{array}\right]
\end{aligned}
$$

Consider a matrix $H \in \Re^{m \times\left(n+n_{c}\right)}$, and define the following polyhedral set:

$$
\mathcal{S}=\left\{\xi\left(t_{k}\right) \in \Re^{n+n_{c}} ;\left|\left(\mathbb{K}_{(i)}-H_{(i)}\right) \xi\left(t_{k}\right)\right| \leq u_{0_{(i)}}\right\}
$$

where $\mathbb{K}_{(i)}, H_{(i)}$ denote the $i^{\text {th }}$ row of matrices $\mathbb{K}$ and $H$, respectively.

Before ending this section, we give the following preliminary results, which will be used subsequently.

Definition 1. [20] The delta operator system (2) is asymptotically stable, if the following condition holds:

$$
\begin{aligned}
V\left(x\left(t_{k}\right)\right) & \geq 0 \text { with equality if and only if } x\left(t_{k}\right)=0 \\
\delta V\left(t_{k}\right) & =\left[V\left(t_{k}+T\right)-V\left(t_{k}\right)\right] / T<0
\end{aligned}
$$

where $V\left(t_{k}\right)$ is a Lyapunov functional in the delta domain.

Lemma 1. [21] If $\xi(t) \in \mathcal{S}$, then the following inequality holds for any positive diagonal matrix $L \in \Re^{m \times m}$ :

$$
\psi^{T}\left(\mathbb{K} \xi\left(t_{k}\right)\right) L\left[\psi\left(\mathbb{K} \xi\left(t_{k}\right)\right)-H \xi\left(t_{k}\right)\right] \leq 0
$$

Lemma 2. [22] For a given symmetric positive definite matrix $M \in \mathbb{R}^{n \times n}$, scalars $0 \leq \sigma_{1} \leq \sigma_{2}$, and vector function $\lambda:\left[-\sigma_{1},-\sigma_{2}\right] \rightarrow \mathbb{R}^{n}$, such that the following sum is well defined, then:

$$
-\left(\sigma_{2}-\sigma_{1}\right) \Sigma_{i=k-\rho_{2}}^{k-\rho_{1}-1} \lambda^{T}\left(t_{k}\right) M \lambda\left(t_{k}\right) \leq-\left[\begin{array}{l}
v_{1} \\
v_{2}
\end{array}\right]^{T}\left[\begin{array}{cc}
M & 0 \\
0 & 3 M
\end{array}\right]\left[\begin{array}{l}
v_{1} \\
v_{2}
\end{array}\right]
$$

where

$\lambda\left(t_{k}\right)=\delta \xi\left(t_{k}\right)$ 
$v_{1}=\xi\left(t_{k}-\sigma_{2}\right)-\xi\left(t_{k}-\sigma_{1}\right)$

$v_{2}=\xi\left(t_{k}-\sigma_{1}\right)+\xi\left(t_{k}-\sigma_{2}\right)-\frac{2}{\sigma_{2}-\sigma_{1}+1} \sum_{i=k-\sigma_{2}}^{k-\sigma_{1}} \xi\left(t_{i}\right)$.

Lemma 3. [9] Consider an interconnected system with two subsystems $\left(S_{1}\right)$ and $\left(S_{2}\right)$ :

$$
\begin{aligned}
\left(S_{1}\right): y\left(t_{k}\right) & =G \omega\left(t_{k}\right) \\
\left(S_{2}\right): \omega\left(t_{k}\right) & =\Delta y\left(t_{k}\right)
\end{aligned}
$$

where the forward system $\left(S_{1}\right)$ is known and the feedback system $\left(S_{2}\right)$ is unknown and time-varying, and assume that $S_{1}$ is internally stable. The closed-loop system composed by $\left(S_{1}\right)$ and $\left(S_{2}\right)$ is robustly asymptotically stable for all $\Delta \in \mathcal{D} \triangleq\left\{\Delta:\|\Delta\|_{\infty} \leq 1\right\}$, if there exist matrices $\left\{T_{\omega}, T_{z}\right\} \in \mathbb{T}$ with: $\mathbb{T} \triangleq\left\{\left\{T_{\omega}, T_{z}\right\} \in \mathbb{R}^{y \times y} \times \mathbb{R}^{z \times z}, T_{\omega}, T_{y}\right.$ non-singular,

$$
\left.\left\|T_{\omega} \circ \Delta \circ T_{y}^{-1}\right\|_{\infty} \leq 1\right\}
$$

such that the following SSGcondition holds:

$$
\left\|T_{y} \circ G \circ T_{\omega}^{-1}\right\|_{\infty} \leq 1 .
$$

Finally, for a scalar $\mu>0$, the ellipsoid $E\left(P, \mu^{-1}\right)$ is given as follows,

$$
E\left(P, \mu^{-1}\right)=\left\{\xi\left(t_{k}\right) \in \Re^{n+n_{c}} ; \quad \xi^{T}\left(t_{k}\right) P \xi\left(t_{k}\right) \leq \mu^{-1}\right\}
$$

Furthermore, we will derive an estimate $\mathcal{D}$ of the domain of initial conditions as,

$$
\mathcal{D}=\left\{\varphi\left(t_{k}\right),-r_{h 2}<t_{k}<0:\left\|\varphi\left(t_{k}\right)\right\| \leq d_{e}\right\}
$$

Remark 2. In computer science and cybernetics, the term "discrete-time delta operator" $\delta$ is generally taken to mean a difference operator,

$$
\delta f\left(t_{k}\right)=\frac{f\left(t_{k}-T\right)-f\left(t_{k}\right)}{T}
$$

the Euler approximation of the usual derivative with a discrete sample time T. The delta-formulation obtains a significant number of numerical advantages compared to the shift-operator at fast sampling. In fact, for fast sampling, the standard forward-shift representation of a discrete-time system becomes extremely sensitive to round-off errors. In addition, it has been demonstrated $[20,23,24]$ that the implementation of delta controllers is significantly less sensitive to round-off errors, round-off noise, and limit cycles than the implementation of the more standard z-domain controllers at a high sampling rate. Furthermore, the stability of delta operator systems by the Lyapunov approach is based on Definition 1 and has been studied by many researchers; see for instance $[20,24]$ for more details on the conditions that must respect the Lyapunov function.

\section{Main Results}

In this section, we will rewrite the system in (6) as a combination of two subsystems $S_{1}$ and $S_{2}$. Therefore, the SSG theorem will be applied to drive the condition for the asymptotic stability of (6). We emphasize here that Lemma 3 serves as a tool for analyzing the stability of any system that can be transformed into a feedback interconnection formulation $S_{1}$ and $S_{2}$, in which we are interested in studying only the stability of the forward subsystem $S_{1}$ since the feedback subsystem $S_{2}$ can be easily normalized, consequently verifying the SSG theorem. It was shown in the literature $[5,6]$ that the use of this transformation and the SSG theorem leads to less restrictive results than other approaches. Here, we used this formulation with the anti-windup strategy, which represents the main novelty in this paper. 
New Model Reformulation

In order to use the input-output approach (SSG theorem), a key solution is to approximate the delay term properly so that the approximation error is as small as possible. In the literature, many authors have tried to find such approximation. The work in $[25,26]$ used a single-term approximation, and two-delay-term approximation was used in [27]. In [6,11], three delay terms were used, and it was shown that the three-delay-term approximation leads to less conservative results than one and two terms. Here, we use the three-term approximation, so we express $\xi\left(t_{k}-r_{h}\right)$ as follows:

$$
\xi\left(t_{k}-r_{h}\right)=\frac{1}{3}\left[\xi\left(t_{k}-r_{h 1}\right)+\xi\left(t_{k}-r_{h a}\right)+\xi\left(t_{k}-r_{h 2}\right)\right]+\frac{r_{h 12}}{3} \tilde{w}\left(t_{k}\right)
$$

where $\xi\left(t_{k}-r_{h 1}\right), \xi\left(t_{k}-r_{h a}\right)$ and $\xi\left(t_{k}-r_{h 2}\right)$ are utilized to approximate $\xi\left(t_{k}-r_{h}\right)$, and $\frac{r_{h 12}}{3} \tilde{w}\left(t_{k}\right)$ represents the approximation error.

Combining (6) with (10), we get the following,

$$
\delta \xi\left(t_{k}\right)=\mathbb{A} \xi\left(t_{k}\right)+\frac{1}{3} \mathbb{A}_{d}\left[\xi\left(t_{k}-r_{h 1}\right)+\xi\left(t_{k}-r_{h a}\right)+\xi\left(t_{k}-r_{h 2}\right)\right]+\frac{r_{h 12}}{3} \mathbb{A}_{d} \tilde{w}\left(t_{k}\right)-\left(\mathbb{B}+\mathbb{R} E_{c}\right) \psi\left(\mathbb{K} \xi\left(t_{k}\right)\right) .
$$

By defining $y\left(t_{k}\right)=\delta \xi\left(t_{k}\right)$, the closed-loop system (6) can be expressed in the form of forward and feedback interconnection systems $\left(S_{1}\right)$ and $\left(S_{2}\right)$ as follows,

$$
\begin{gathered}
\left(S_{1}\right):\left[\begin{array}{c}
\delta \xi\left(t_{k}\right) \\
y\left(t_{k}\right)
\end{array}\right]=\left[\begin{array}{cc}
\mathrm{Y}_{1} & \frac{r_{h 12}}{3} \mathbb{A}_{d} \\
\mathrm{Y}_{1} & \frac{r_{h 12}}{3} \mathbb{A}_{d}
\end{array}\right]\left[\begin{array}{c}
\zeta\left(t_{k}\right) \\
\widetilde{\omega}\left(t_{k}\right)
\end{array}\right] \\
\left(S_{2}\right): \omega\left(t_{k}\right)=\Delta y\left(t_{k}\right)
\end{gathered}
$$

where:

$$
\begin{aligned}
\mathrm{Y}_{1} & =\left[\begin{array}{lllll}
\mathbb{A} & \frac{1}{3} \mathbb{A}_{d} & \frac{1}{3} \mathbb{A}_{d} & \frac{1}{3} \mathbb{A}_{d} & -\left(\mathbb{B}+\mathbb{R} E_{c}\right)
\end{array}\right] \\
\zeta^{T}\left(t_{k}\right) & =\left[\begin{array}{lllll}
\xi^{T}\left(t_{k}\right) & \xi^{T}\left(t_{k}-r_{h 1}\right) & \xi^{T}\left(t_{k}-r_{h a}\right) & \xi^{T}\left(t_{k}-r_{h 2}\right) & \psi^{T}\left(\mathbb{K} \xi\left(t_{k}\right)\right)
\end{array}\right]
\end{aligned}
$$

Concerning the approximation error, we will use the same steps of the work [11]. Before moving on, the following lemma guarantees that the $l_{2}$ induced norm of $\Delta$ is bounded by one.

Lemma 4. Mapping $\Delta: y \rightarrow \omega$ of the feedback subsystem $S_{2}$ satisfies $\|\Delta\| \leq 1$.

The proof of Lemma 4 follows the same process as in the paper [11], and introducing a factor of three due to the Cauchy-Schwartz inequality results in $\tilde{\omega}=\frac{3}{\sqrt{2}} \omega$

The following theorem presents the criterion of the stability for the delta operator system (6).

Theorem 1. For given scalars $\gamma, \mu$, the system (6) is asymptotically stable, if there exist symmetric positive definite matrices $X, \tilde{P}, \tilde{S}, \tilde{Q}_{j}(j=1,2,3), \tilde{R}_{i}(i=1,2)$, and appropriately-sized matrices $V_{c}, L, W$ such that:

$$
\begin{gathered}
{\left[\begin{array}{ccc}
\tilde{\Omega}_{1} & \tilde{\Omega}_{2}^{T} & \tilde{\Omega}_{3}^{T} \\
* & -12 \tilde{R}_{1} & 0 \\
* & * & -12 \tilde{R}_{2}
\end{array}\right]<0} \\
{\left[\begin{array}{cc}
\tilde{P} & X \mathbb{K}_{i}^{T}-W_{i}^{T} \\
* & \mu u_{o_{(i)}}^{2}
\end{array}\right] \geq 0 \quad i=1, \ldots, m}
\end{gathered}
$$


where:

$$
\begin{aligned}
& \tilde{\Omega}_{1}=\left[\begin{array}{ccc}
\tilde{\Omega}_{11} & \tilde{P}+\gamma \mathbb{A} X^{T}-X & \gamma \tilde{\Omega}_{13} \\
* & \tilde{\Omega}_{12} & \tilde{\Omega}_{13}+\tilde{\Omega}_{14} \\
* & * & -\tilde{\Omega}_{15}
\end{array}\right] \\
& \tilde{\Omega}_{11}=T \tilde{P}+\left(r_{h 1} T\right)^{2} \tilde{R}_{1}+\left(r_{h 2} T\right)^{2} \tilde{R}_{2}+\tilde{S}-\gamma X-\gamma X^{T} \\
& \tilde{\Omega}_{12}=\tilde{Q}_{1}+\tilde{Q}_{2}+\tilde{Q}_{3}-4 \tilde{R}_{1}-4 \tilde{R}_{2}+\mathbb{A} X^{T}+X \mathbb{A}^{T} \\
& \tilde{\Omega}_{13}=\left[\frac{1}{3} \mathbb{A}_{d} X^{T} \frac{1}{3} \mathbb{A}_{d} X^{T} \frac{1}{3} \mathbb{A}_{d} X^{T} \frac{1}{3} r_{h 12} \mathbb{A}_{d} X^{T}-\mathbb{B} L-\mathbb{R} V_{c}\right] \\
& \tilde{\Omega}_{14}=\left[\begin{array}{lllll}
-2 \tilde{R}_{1} & 0 & -2 \tilde{R}_{2} & 0 & W^{T}
\end{array}\right] \\
& \tilde{\Omega}_{15}=\operatorname{diag}\left\{\tilde{Q}_{1}+4 \tilde{R}_{1}, \tilde{Q}_{3}, \tilde{Q}_{2}+4 \tilde{R}_{2}, \frac{2}{9} \tilde{S}, L+L^{T}\right\} \\
& \tilde{\Omega}_{2}=\left[\begin{array}{llll}
0 & 6 \tilde{R}_{1}^{T} & 6 \tilde{R}_{1}^{T} & 0_{n \times 4 n}
\end{array}\right] \\
& \tilde{\Omega}_{3}=\left[\begin{array}{lllll}
0 & 6 \tilde{R}_{2}^{T} & 0_{2 n} & 6 \tilde{R}_{2}^{T} & 0_{2 n}
\end{array}\right] \text {. }
\end{aligned}
$$

Moreover, the anti-windup compensation gain is given by $E_{c}=V_{c} L^{-1}$ and guarantees that the trajectories of the closed-loop system are bounded for every initial condition in the following ball,

$$
d_{e}^{2}\left\{\bar{\lambda}\left(X^{-1} \tilde{P} X^{-T}\right)+T\left(r_{h 1}+r_{h 2}\right) \sum_{l=1}^{3} \bar{\lambda}\left(X^{-1} \tilde{Q}_{l} X^{-T}\right)+4 T^{3} \sum_{l=1}^{2} \bar{\lambda}\left(X^{-1} \tilde{R}_{l} X^{-T}\right)\right\} \leq \mu^{-1} .
$$

Proof. Consider the following Lyapunov-Krasovskii functional (LKF),

$$
V\left(t_{k}\right)=V_{1}\left(t_{k}\right)+V_{2}\left(t_{k}\right)+V_{3}\left(t_{k}\right)
$$

where:

$$
\begin{aligned}
& V_{1}\left(t_{k}\right)=\xi^{T}\left(t_{k}\right) P \xi\left(t_{k}\right) \\
& V_{2}\left(t_{k}\right)=T \sum_{l=1}^{3} \sum_{i=k-h_{l}}^{k-1} \xi^{T}\left(t_{i}\right) Q_{l} \xi\left(t_{i}\right), h_{3}=h_{a} \\
& V_{3}\left(t_{k}\right)=T^{3} \sum_{l=1}^{2} \sum_{i=-h_{l}}^{-1} \sum_{j=k+i}^{k-1} r_{l} y^{T}\left(t_{j}\right) R_{l} y\left(t_{j}\right) .
\end{aligned}
$$

Taking the delta operator manipulation of $V_{1}\left(t_{k}\right)$ and using Lemma 1 in [9], we obtain:

$$
\delta V_{1}\left(t_{k}\right)=2 \xi^{T}\left(t_{k}\right) P \delta \xi\left(t_{k}\right)+T \delta \xi^{T}\left(t_{k}\right) P \delta \xi\left(t_{k}\right)
$$

We keep $\delta \xi\left(t_{k}\right)$ in $T \delta \xi\left(t_{k}\right) P \delta \xi\left(t_{k}\right)$, and we change $\delta \xi\left(t_{k}\right)$ according to the expression in $\left(S_{1}\right)$. The forward difference of $V_{2}\left(t_{k}\right)$ can be directly calculated by applying Definition 1 .

$$
\begin{aligned}
\delta V_{2}\left(t_{k}\right)= & \xi\left(t_{k}\right)^{T}\left(Q_{1}+Q_{2}+Q_{3}\right) \xi\left(t_{k}\right)-\xi^{T}\left(t_{k}-r_{h 1}\right) Q_{1}\left(t_{k}-r_{h 1}\right) \\
& -\xi^{T}\left(t_{k}-r_{h 2}\right) Q_{2} \xi\left(t_{k}-r_{h 2}\right) \\
& -\xi^{T}\left(t_{k}-r_{h a}\right) Q_{3} \xi\left(t_{k}-r_{h a}\right)
\end{aligned}
$$

Based on the Wirtinger-based inequality, we obtain:

$$
\delta V_{3} \leq y^{T}\left(t_{k}\right)\left(r_{h 1}^{2} T^{2} R_{1}+r_{h 2}^{2} T^{2} R_{2}\right) y\left(t_{k}\right)+\beta^{T} v_{1} \beta+\alpha^{T} v_{2} \alpha
$$


where

$\beta^{T}=\left[\xi^{T}\left(t_{k}\right) \quad \xi^{T}\left(t_{k}-r_{h 1}\right) \frac{1}{h_{1}+1} \sum_{i=k-h_{1}}^{k} \xi^{T}\left(t_{i}\right)\right], \quad \alpha^{T}=\left[\xi^{T}\left(t_{k}\right) \quad \xi^{T}\left(t_{k}-r_{h 2}\right) \frac{1}{h_{2}+1} \sum_{i=k-h_{2}}^{k} \xi^{T}\left(t_{i}\right)\right]$

$v_{1}=\left[\begin{array}{ccc}-4 R_{1} & -2 R_{1} & 6 R_{1} \\ * & -4 R_{1} & 6 R_{1} \\ * & * & -12 R_{1}\end{array}\right] \quad, v_{2}=\left[\begin{array}{ccc}-4 R_{2} & -2 R_{2} & 6 R_{2} \\ * & -4 R_{2} & 6 R_{2} \\ * & * & -12 R_{2}\end{array}\right]$

Using the free weighting matrix technique, for appropriate matrices $N_{1}$ and $N_{2}$, we have that:

$$
\begin{aligned}
0= & 2\left[\xi^{T}\left(t_{k}\right) N_{1}+\delta \xi^{T}\left(t_{k}\right) N_{2}\right]\left[-\delta \xi\left(t_{k}\right)+\mathbb{A} \xi\left(t_{k}\right)+\frac{\mathbb{A}_{d}}{3} x\left(t_{k}-r_{h 1}\right)\right. \\
& +\frac{\mathbb{A}_{d}}{3} x\left(t_{k}-r_{h a}\right)+\frac{\mathbb{A}_{d}}{3} x\left(t_{k}-r_{h 2}\right) \\
& \left.+\frac{r_{h 12}}{3} \mathbb{A}_{d} \widetilde{\omega}\left(t_{k}\right)-\left(\mathbb{B}+\mathbb{R} E_{c}\right) \psi\left(\mathbb{K} \xi\left(t_{k}\right)\right)\right]
\end{aligned}
$$

Let $S=D^{T} D$, and following $[5,6,11]$, define,

$$
J\left(t_{k}\right)=y^{T}\left(t_{k}\right) S y\left(t_{k}\right)-\omega^{T}\left(t_{k}\right) S \omega\left(t_{k}\right)
$$

and $J=\sum_{k=0}^{\infty} J\left(t_{k}\right)$.

Combining Equations (16)-(19), it follows that,

$$
\sum_{k=0}^{\infty}\left[J\left(t_{k}\right)+\delta V\left(t_{k}\right)\right] \leq \sum_{k=0}^{\infty} \zeta^{T}\left(t_{k}\right) \Omega \zeta\left(t_{k}\right)
$$

where,

$$
\begin{aligned}
& \zeta^{T}=\left[\begin{array}{llllll}
\delta \xi^{T}\left(t_{k}\right) & \xi^{T}\left(t_{k}\right) & \xi^{T}\left(t_{k}-r_{h 1}\right) & \xi^{T}\left(t_{k}-r_{h a}\right) & \xi^{T}\left(t_{k}-r_{h 2}\right) & \widetilde{\omega}^{T}\left(t_{k}\right)
\end{array}\right. \\
& \left.\psi^{T}\left(\mathbb{K} \xi\left(t_{k}\right)\right) \frac{1}{h_{1}+1} \sum_{i=k-h_{1}}^{k} \xi^{T}\left(t_{i}\right) \frac{1}{h_{2}+1} \sum_{i=k-h_{2}}^{k} \xi^{T}\left(t_{i}\right)\right] \\
& \Omega=\left[\begin{array}{ccc}
\Omega_{1} & \Omega_{2}^{T} & \Omega_{3}^{T} \\
* & -12 R_{1} & 0 \\
* & * & -12 R_{2}
\end{array}\right],
\end{aligned}
$$

and where the elements of $\Omega$ are given by,

$$
\begin{aligned}
& \Omega_{1}=\left[\begin{array}{ccc}
\Omega_{11} & P+N_{2} \mathbb{A}-N_{1}^{T} & N_{2} \Omega_{13} \\
* & \Omega_{12} & N_{1} \Omega_{13}+\Omega_{14} \\
* & * & -\Omega_{15}
\end{array}\right], \\
& \Omega_{2}=\left[\begin{array}{llll}
0 & 6 R_{1}^{T} & 6 R_{1}^{T} & 0_{n \times 4 n}
\end{array}\right], \quad \Omega_{3}=\left[\begin{array}{llllll}
0 & 6 R_{2}^{T} & 0_{2 n} & 6 R_{2}^{T} & 0_{2 n}
\end{array}\right] \\
& \Omega_{11}=T P+S+T^{2}\left(r_{h 1}^{2} R_{1}+r_{h 2}^{2} R_{2}\right)-N_{2}-N_{2}^{T} \\
& \Omega_{12}=Q_{1}+Q_{2}+Q_{3}-4 R_{1}-4 R_{2}+N_{1} \mathbb{A}+\mathbb{A}^{T} N_{1}^{T} \\
& \Omega_{13}=\left[\frac{1}{3} \mathbb{A}_{d} \frac{1}{3} \mathbb{A}_{d} \frac{1}{3} \mathbb{A}_{d} \frac{1}{3} r_{h 12} \mathbb{A}_{d}-\left(\mathbb{B}+\mathbb{R} E_{c}\right)\right] \\
& \Omega_{14}=\left[\begin{array}{lllll}
-2 R_{1} & 0 & -2 R_{2} & 0 & H^{T} T_{0}^{T}
\end{array}\right] \\
& \Omega_{15}=\operatorname{diag}\left\{Q_{1}+4 R_{1}, Q_{3}, Q_{2}+4 R_{2}, \frac{2}{9} S, T_{0}+T_{0}^{T}\right\} \text {. }
\end{aligned}
$$


Multiplying (22) by $\operatorname{diag}\left\{N_{1}^{-1}, N_{1}^{-1}, N_{1}^{-1}, N_{1}^{-1}, N_{1}^{-1}, N_{1}^{-1}, T_{0}^{-1}, N_{1}^{-1}, N_{1}^{-1}\right\}$ from the left and its transpose on the right and by defining $N_{1}^{-1}=X, N_{2}=\gamma N_{1}, V_{c}=E_{c} T_{T}^{-1}, \tilde{P}=X P X^{T}, \tilde{R}_{i}=X R_{i} X^{T}, i=$ $1,2, \tilde{Q}_{j}=X Q_{j} X^{T}, j=1,2,3, \tilde{S}=X S X^{T}, W=H X^{T}, L=T_{0}^{-1}$, we obtain the LMI (12). Since (12) holds, we can conclude that,

$$
\sum_{k=0}^{\infty}\left[J\left(t_{k}+\delta V\left(t_{k}\right)\right]<0\right.
$$

Under zero initial condition, $V(0)=0$, we have,

$$
\begin{aligned}
\sum_{k=0}^{\infty}\left[J\left(t_{k}\right)+\delta V\left(t_{k}\right)\right] & =J+V(\infty)-V(0) \\
& =J+V(\infty)<0 .
\end{aligned}
$$

This implies that $J<0$. This implies that there exist invertible matrices such that the system $\left(S_{1}\right)$ satisfies (9).

From (23), it follows that $\delta V\left(t_{k}\right)<0$ when the disturbance input of system $\left(S_{1}\right)$ is null, which implies the asymptotic stability of $\left(S_{1}\right)$.

On the other side, the LMI (13) ensures that $\forall \xi \in E\left(P, \mu^{-1}\right), \xi \in \mathcal{S}$. In fact, $E\left(P, \mu^{-1}\right) \subset \mathcal{S}$ can be guaranteed by the next condition,

$$
\left[\begin{array}{cc}
P & \mathbb{K}_{(i)}^{T}-H_{(i)}^{T} \\
* & \mu u_{0_{(i)}}^{2}
\end{array}\right] \geq 0 \quad i=1, \ldots, m .
$$

Pre- and post-multiplying (24) by $\operatorname{diag}\left\{N^{-1}, I\right\}$ will result in the LMI (13).

Furthermore, from $\delta V\left(t_{k}\right)<0$, it follows that $V\left(t_{k}\right) \leq V(0)$, and thus, we obtain,

$$
V(0) \leq V_{1}(0)+V_{2}(0)+V_{3}(0) \leq\left(\bar{\lambda}(P)+T\left(r_{h 1}+r_{h 2}\right) \sum_{l=1}^{3} \bar{\lambda}\left(Q_{l}\right)+4 T^{3} \sum_{l=1}^{2} \bar{\lambda}\left(R_{l}\right)\right)\|\varphi\|^{2}=\kappa
$$

Therefore, we have:

$$
\xi^{T}\left(t_{k}\right) P \xi\left(t_{k}\right) \leq V\left(t_{k}\right) \leq V(0) \leq \kappa \leq \mu^{-1}
$$

Finally, we obtain (14). Then, the inequality (14) guarantees that the trajectories of $\xi\left(t_{k}\right)$ remain within $\mathcal{D}$ for all initial functions $\varphi\left(t_{k}\right)$, and this completes the proof.

\section{Anti-Windup Optimization}

Since (14) is nonlinear, it cannot be solved directly by applying the useful numerical tools (such as the LMI-toolbox of MATLAB). It follows that it is difficult to come up with a solution such that the domain of initial conditions is the largest possible. Thus, to overcome such a problem, we propose an optimization problem under which the nonlinear conditions are transformed to matrix inequalities, which can be easily solved. We let $X^{-1} \tilde{P} X^{-T} \leq \sigma_{P} I, X^{-1} \tilde{Q}_{l} X^{-T} \leq \sigma_{Q_{l}} I, X^{-1} \tilde{R}_{l} X^{-T} \leq \sigma_{R_{l}} I$. With this aim, consider the following auxiliary LMIs where $\hat{X}=X^{-1}, \hat{P}=\tilde{P}^{-1}, \hat{Q}_{l}=\tilde{Q}_{l}^{-1},(l=1,2,3)$, and $\hat{R}_{l}=$ $\tilde{R}_{l}^{-1},(l=1,2)$ :

$$
\left[\begin{array}{cc}
\sigma_{P} I & \hat{X} \\
\hat{X}^{T} & \hat{P}
\end{array}\right] \geq 0, \quad\left[\begin{array}{cc}
\sigma_{Q_{I} I} & \hat{X} \\
\hat{X}^{T} & \hat{Q}_{l}
\end{array}\right] \geq 0, \quad\left[\begin{array}{cc}
\sigma_{R_{l} I} & \hat{X} \\
\hat{X}^{T} & \hat{R}_{l}
\end{array}\right] \geq 0
$$

The condition (14) is verified if the following LMI is satisfied,

$$
d_{e}^{2}\left\{\sigma_{P}+T\left(r_{h 1}+r_{h 2}\right) \sum_{l=1}^{3} \sigma_{Q_{l}}+4 T^{3} \sum_{l=1}^{2} \sigma_{R_{l}}\right\} \leq \mu^{-1} .
$$


Then, as in $[28,29]$, we formulate a feasibility problem as follows, $\min \operatorname{tr}\left(\tilde{P} \hat{P}+\tilde{Q}_{l} \hat{Q}_{l}+\tilde{R}_{l} \hat{R}_{l}+\left(X+X^{T}\right)\left(\hat{X}+\hat{X}^{T}\right)\right)$ subject to (12), (13), (25), and (26),

$$
\left[\begin{array}{cc}
\tilde{P} & * \\
I & \hat{P}
\end{array}\right] \geq 0,\left[\begin{array}{cc}
\tilde{Q}_{l} & * \\
I & \hat{Q}_{l}
\end{array}\right] \geq 0,\left[\begin{array}{cc}
\tilde{R}_{l} & * \\
I & \hat{R}_{l}
\end{array}\right] \geq 0,\left[\begin{array}{cc}
X+X^{T} & * \\
I & \hat{X}+\hat{X}^{T}
\end{array}\right] \geq 0 .
$$

Based on the above conditions, the proposed controller can be designed for given $r_{h 1}$ and $r_{h 2}$ by utilizing the following cone complementarity algorithm.

Step 1 For given $r_{h 1}, r_{h 2}$, and $\mu$, fix a sufficiently large $d_{e}$ such that the constrained minimization (27) is feasible. Then, set $\left(\tilde{P}, \hat{P}, \tilde{Q}_{l}, \hat{Q}_{l}, \tilde{R}_{l}, \hat{R}_{l}, X, \hat{X}, \sigma_{P}, \sigma_{Q_{l}}, \sigma_{R_{l}}\right)_{0}=$ $\left(\tilde{P}, \hat{P}, \tilde{Q}_{l}, \hat{Q}_{l}, \tilde{R}_{l}, \hat{R}_{l}, X, \hat{X}, \sigma_{P}, \sigma_{Q_{l}}, \sigma_{R_{l}}\right)$. Fix a sufficiently small $D_{e}$, and set $d_{e}=d_{e}+D_{e}$.

Step 2 Solve the following LMI minimization problem :

$\min \operatorname{tr}\left(\tilde{P} \hat{P}_{0}+\tilde{Q}_{l} \hat{Q}_{l_{0}}+\tilde{R}_{l} \hat{R}_{l_{0}}+\left(X+X^{T}\right)\left(\hat{X}_{0}+\hat{X}_{0}^{T}\right)+\tilde{P}_{0} \hat{P}+\tilde{Q}_{l_{0}} \hat{Q}_{l}+\tilde{R}_{l_{0}} \hat{R}_{l}+\left(X_{0}+X_{0}^{T}\right)\left(\hat{X}+\hat{X}^{T}\right)\right)$ subject to LMIs in (27).

Step 3 Substitute the new matrix variables into (27). If the result is feasible, then set $d_{e}=d_{e}+D_{e}$ and the new solution as $\left(\tilde{P}, \hat{P}, \tilde{Q}_{l}, \hat{Q}_{l}, \tilde{R}_{l}, \hat{R}_{l}, X, \hat{X}, \sigma_{P}, \sigma_{Q_{l}}, \sigma_{R_{l}}\right)_{0}$, and repeat Step 2; otherwise, $d_{e}=d_{e}-D_{e}$ is the required estimate: Stop.

Remark 3. The initial value of $d_{e}$ in Step 1 of the above algorithm can always be found by solving (14) when the conditions (12) and (13) are feasible. The condition (27) includes the conditions (12) and (13), and the nonlinear condition (14) is transformed into linear matrix inequalities. Furthermore, the incremental step $D_{e}$ is generally chosen sufficiently small to guarantee the best accuracy. Note that inequalities (12) and (13) can be easily solved by using the LMI toolbox of MATLAB or Scilab.

Remark 4. The determination of the basin of attraction for delta operator systems under input limitations has received little attention; see $[17,18,30,31]$. All the cited references used the polytopic description of the saturation nonlinearity, based on the convex hull representation. In [19], nested actuator saturation was studied based on the convex hull representation of the saturation nonlinearity. However, until now, the anti-windup approach was not considered in the literature for the delta operator system. Historically, the "windup" term is linked to the effects of actuator limitations in control input that contains the integral action with extra charge, which produces side effects on the transient response of the system, like slow convergence to the equilibrium point and oscillation. To overcome the undesirable effects caused by the saturation, we can add another control-loop, that we call anti-windup compensation for the closed-loop system. In this paper, this approach is used to guarantee both asymptotic stability of the delta operator and an estimate of the domain of attraction.

Remark 5. It should be mentioned that all the existing delta operator systems with control saturation (see for example $[17,18])$ do not investigate the anti-windup approach. However, in this paper, we utilize this approach to guarantee the system stability in an estimate of the domain of attraction for the delta operator system with input limitations. Therefore, the proposed strategy of control is a new approach for controlling delta operator systems and gives insight into the design of controllers for such systems.

\section{Numerical Examples}

In this section, we present two numerical examples to validate the correctness and superiority of the designed control scheme.

Example 1. In order to evaluate our results and due to the fact that there are no results in the literature that deal with the ant-windup for delta operator systems, in this example, we will compare our approach with a literature 
result studying the stability of delta operator systems by using the polytopic representation of the saturation function. We consider a delta operator system with the following matrices [17],

$$
A=\left[\begin{array}{ll}
0.0500 & 1.0017 \\
1.0017 & 0.0500
\end{array}\right], \quad B=\left[\begin{array}{l}
0.2502 \\
5.0083
\end{array}\right]
$$

The dynamic controller has the following parameters,

$$
A_{c}=\left[\begin{array}{cc}
-4.4294 & -19.7165 \\
-21.2406 & -149.0227
\end{array}\right], B_{c}=\left[\begin{array}{c}
-2.5278 \\
-23.4050
\end{array}\right], C_{c}=\left[\begin{array}{ll}
0.8459 & 10.8186
\end{array}\right], D_{c}=0
$$

By applying the proposed algorithm with $T=0.1$ and $\gamma=0.5$, the estimate of the region of attraction is obtained as $d_{e}=30.9$ and $E_{c}=\left[\begin{array}{ll}-1.2903 & 0.1842\end{array}\right]^{T}$, while the result of [17] is $d_{e}=0.5317$. It can be found that the obtained $d_{e}$ is larger than that obtained $d_{e}$ in [17], which shows the effectiveness of the presented method. The state response of the closed-loop system with initial conditions $x(0)=\left[\begin{array}{ll}1 & -1\end{array}\right]$ is depicted in Figure 1. Obviously, the curves of the state response converge to the equilibrium point.

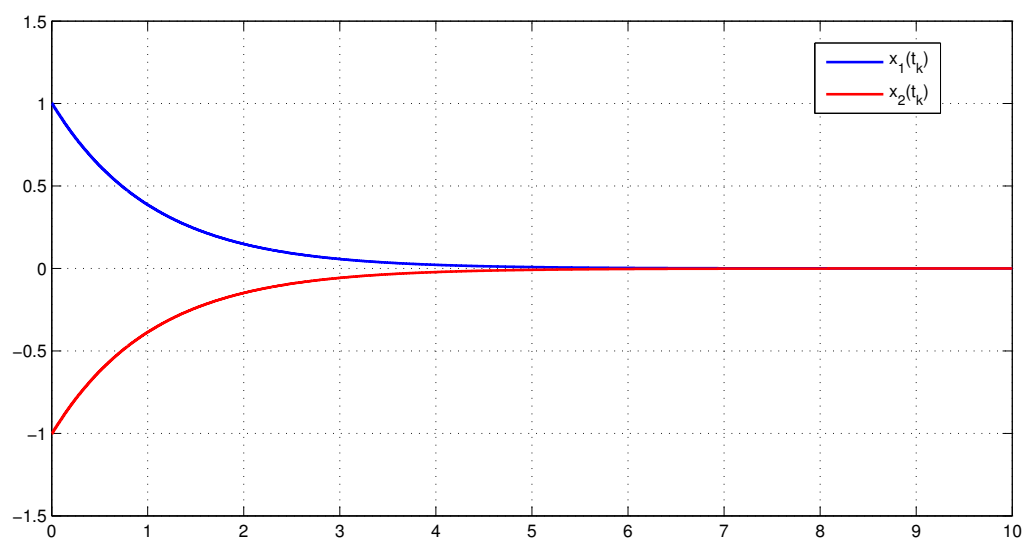

Figure 1. State response of the closed-loop system.

Example 2. Given a delta operator system with:

$$
A=\left[\begin{array}{cc}
1 & 1.5 \\
0.3 & -2
\end{array}\right], \quad A_{d}=\left[\begin{array}{cc}
0 & -1 \\
0 & 0
\end{array}\right], \quad B=\left[\begin{array}{c}
10 \\
0
\end{array}\right], \quad C_{y}=\left[\begin{array}{ll}
5 & 1
\end{array}\right], \quad u_{0}=15
$$

The dynamic controller is given as:

$$
A_{c}=\left[\begin{array}{cc}
-20.2042 & 2.5216 \\
2.1415 & -4.4821
\end{array}\right], B_{c}=\left[\begin{array}{c}
1.9516 \\
-0.0649
\end{array}\right], C_{c}=\left[\begin{array}{ll}
-0.9165 & 0.1091
\end{array}\right], D_{c}=0 .
$$

Applying Theorem 1 with $T=0.01$ and $\gamma=0.7$, we obtain the maximum upper bound $r_{h 2}$ and the corresponding anti-windup gain matrix according to the value of $r_{h 1}$, as listed in the following Table 1. 
Table 1. Calculated upper bound $r_{h 2}$ for different values of $r_{h 1}$.

\begin{tabular}{ccc}
\hline$r_{\boldsymbol{h 1}}$ & $r_{\boldsymbol{h} \mathbf{2}}$ & \multicolumn{2}{c}{$\boldsymbol{E}_{\boldsymbol{c}}$} \\
\hline 1 & 21 & {$\left[\begin{array}{ll}15.0338 & -2.4257\end{array}\right]^{T}$} \\
3 & 23 & {$\left[\begin{array}{ll}14.7327 & -2.2891\end{array}\right]^{T}$} \\
5 & 25 & {$\left[\begin{array}{ll}15.2971 & -2.6786\end{array}\right]^{T}$} \\
7 & 27 & {$\left[\begin{array}{ll}14.3278 & 2.3199\end{array}\right]^{T}$} \\
9 & 29 & {$\left[\begin{array}{ll}14.9153 & -0.2678\end{array}\right]^{T}$} \\
11 & 31 & {$\left[\begin{array}{ll}15.1340 & -0.3745\end{array}\right]^{T}$} \\
\hline
\end{tabular}

Figures 2 and 3 depict the simulation result for state trajectories of the closed-loop system with the anti-windup compensator (for the anti-windup gain corresponding to $r_{h 1}=11$ ) and without the anti-windup compensator, respectively, with the initial condition $x(0)=[3-4]^{T}$. It is seen clearly that the the time response was greatly improved by the usage of the proposed anti-windup compensator.

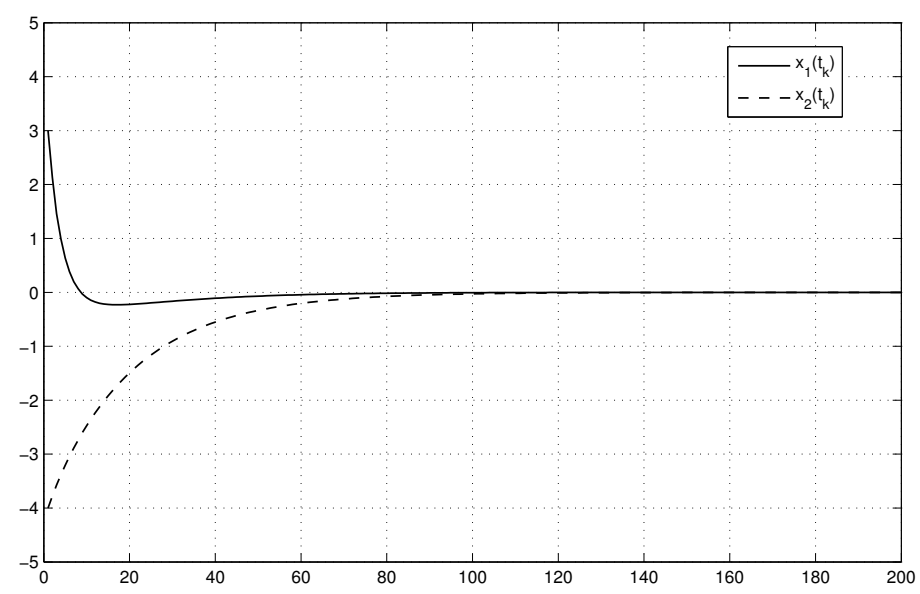

Figure 2. State trajectories of the closed-loop system with anti-windup.

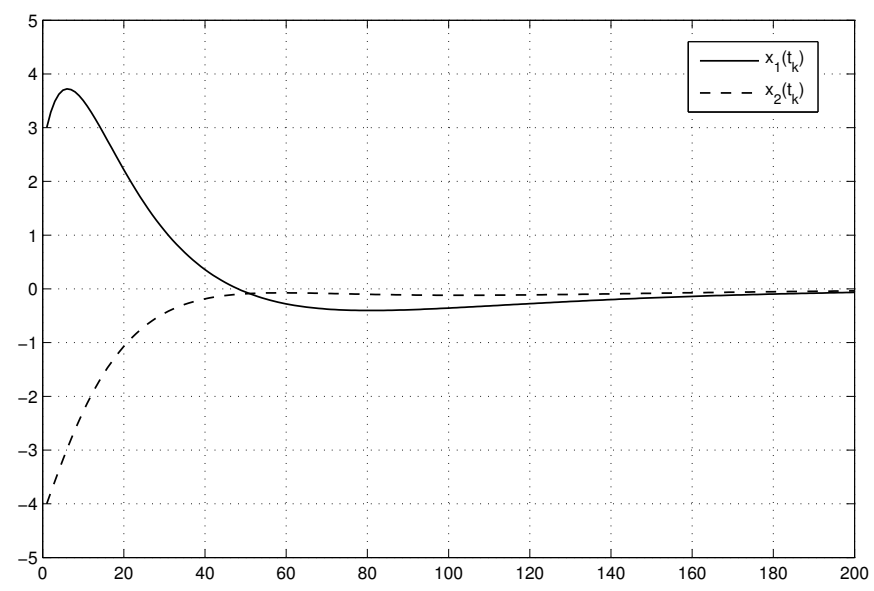

Figure 3. State trajectories of the closed-loop system without anti-windup.

Figures 4 and 5 show the control signal with the anti-windup compensator and without the anti-windup compensator. It can be observed that the time in which the control signal remained saturated was smaller with the anti-windup. 


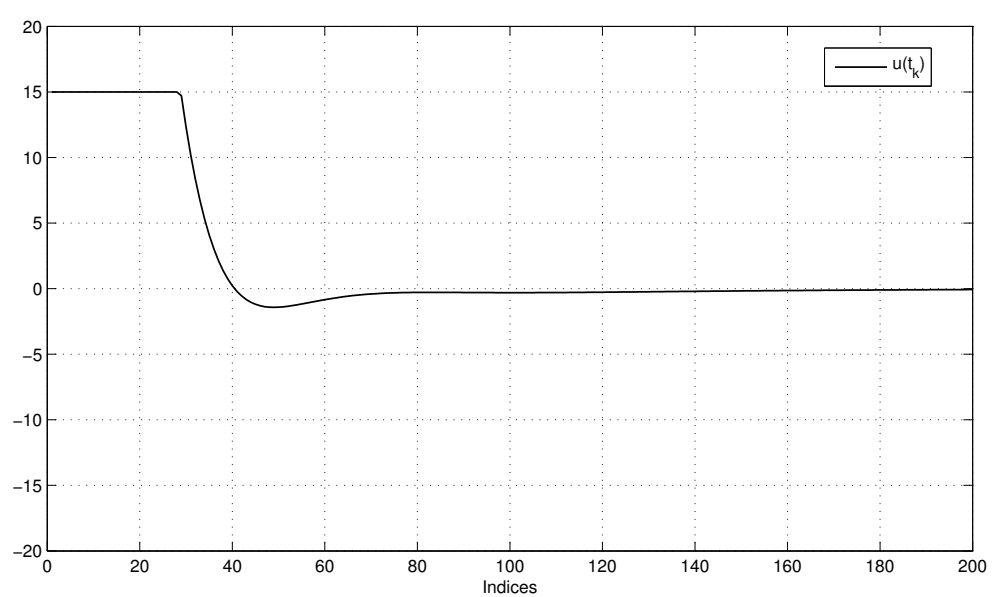

Figure 4. Control input with anti-windup.

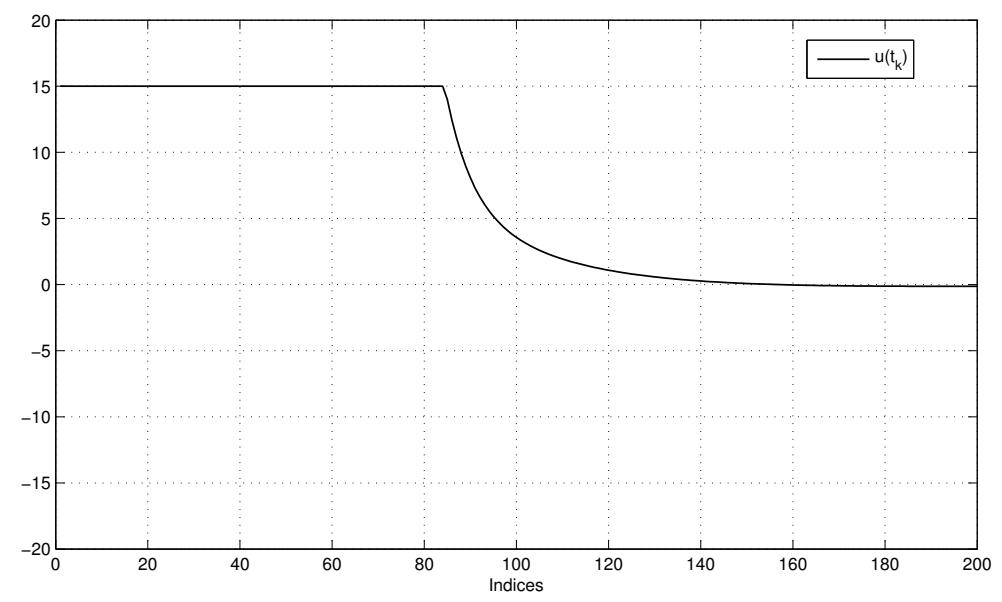

Figure 5. Control input without anti-windup.

\section{Conclusions}

The problem of anti-windup design was investigated for a class of delta operator systems subject to actuator saturation and time-varying delay. By incorporating the SSG theorem, the IO approach, and Wirtinger's integral inequality, a sufficient condition guaranteeing both the stabilization and an estimate of the region of attraction was derived in terms of the LMIs. An optimization procedure was proposed to obtain the maximal domain of initial conditions that ensured the stability of the system. The potential of the proposed strategy was illustrated via two numerical examples. Note that the controller model was given by (3), for which the matrices were assumed to be given. Assuming these matrices are not known and are to be determined requires further development and will be the subject of a future contribution.

Author Contributions: Supervision, E.H.T.; writing, original draft, H.R.; writing, review and editing, O.L.

Funding: This research received no external funding.

Acknowledgments: The authors would like to thank the editor and anonymous reviewers for their many helpful comments and suggestions improved the quality of this paper.

Conflicts of Interest: The authors declare that they have no conflicts of interests. 


\section{References}

1. Ech-charqy, A.; Ouahi, M.; Tissir, E.H. Delay-dependent robust stability criteria for singular time-delay systems by delay-partitioning approach. Int. J. Syst. Sci. 2018, 39, 2957-2967. [CrossRef]

2. He, Y.; Wang, Q.G.; Xie, L.; Lin, C. Further improvement of free weighting matrices technique for systems with time-delay. IEEE Trans. Autom. Control 2007, 52, 293-299. [CrossRef]

3. Tissir, E.H. Delay-dependent robust stability of linear systems with non-commensurate time varying delays. Int. J. Syst. Sci. 2007, 38, 749-757. [CrossRef]

4. Liu, Y.; Li, M. Improved robust stabilization method for linear systems with interval time-varying input delays by using wirtinger inequality. ISA Trans. 2015, 56, 111-122. [CrossRef]

5. Belmfedel Alaoui, S.; Tissir, E.H.; Chaibi, N. Small-Gain Theorem and Finite Frequency Analysis of TCP/AQM System With Time Varing Delay. IET Control Theory Appl. 2019, 13, 1971-1982.

6. El Aiss, H.; Hmamed, A.; El Hajjaji, A. Improved stability and $H_{\infty}$ performance criteria for linear systems with interval time-varying delays via three terms approximation. Int. J. Syst. Sci. 2017, 48, 3450-3458. [CrossRef]

7. Goodwin, G.C.; Leal, R.L.; Mayne, D.Q. Rapprochement between continuous and discrete model refrence adaptive control. Automatica 1986, 22, 199-207. [CrossRef]

8. Mrabti, M.; Hmamed, A. Bounds for the solution of the Lyapunov matrix equation a unified approach. Syst. Control Lett. 1992, 18, 73-81 [CrossRef]

9. Xie, X.; Yin, S.; Gao, H. Asymptotic stability and stabilization of uncertain delta operator systems with time varying delays. IET Control Theory Appl. 2013, 7, 1071-1078. [CrossRef]

10. $\mathrm{Xu}, \mathrm{M}$. Robust $H_{\infty}$ filtering design for Takagi-Sugeno fuzzy systems with time-varying delay via delta operator approach. Adv. Mech. Eng. 2017,1-13. [CrossRef]

11. El Aiss, H.; Rachid, H.; Hmamed, A.; El Hajjaji, A. Approach to delay-dependent robust stability and stabilisation of delta operator systems with time-varying delays. IET Control Theory Appl. 2018, 12, 786-792. [CrossRef]

12. El Fezazi, N.; Tissir, E.H.; Haoussi, F.E.; Alvarez, T.; Tadeo, F. Control Based on Saturated Time-Delay Systems Theory of Mach Number in Wind Tunnels. Circuits Syst. Signal Process 2017. [CrossRef]

13. El Fezazi, N.; Haoussi, F.E.; Tissir, E.H.; Alvarez, T.; Tadeo, F. Robust stabilization using a sampled-data strategy of uncertain neutral state-delayed systems subject to input limitations. Circuits Int. J. Appl. Math. Comput. Sci. 2018, 28, 111-122, . [CrossRef]

14. Lamrabet, O.; Ech-charqy, A.; Tissir, E.H.; El Haoussi, F. Sampled data Control for Takagi-Sugeno Fuzzy Systems with Actuator Saturation. Procedia Comput. Sci. 2019, 148, 448-454. [CrossRef]

15. Lamrabet, O.; Tissir, E.H.; El Haoussi, F. Anti-windup Compensator Synthesis for Sampled-Data Delay Systems. Circuits Syst. Signal Process 2018. [CrossRef]

16. Lamrabet, O.; El Fezazi, N.; El Haoussi, F.; Tissir, E.H. Using Input Delay Approach for Synthesizing an Anti-windup Compensator to AQM in TCP/IP Networks. In Proceedings of the International Conference on Advanced Technologies, Signal and Image Processing, Fez, Morocco, 22-24 May 2017. [CrossRef]

17. Yang, H.; Shi, P.; Li, Z.; Hua, C. Analysis and design for delta operator systems with actuator saturation. Int. J. Control. 2014, 87, 987-999 . [CrossRef]

18. Yang, H.; Li, Z.; Hua, C.; Liu, Z. Stability Analysis of Delta Operator Systems with Actuator Saturation by a Saturation-Dependent Lyapunov Function. Circuits Syst. Signal Process 2014. [CrossRef]

19. Shi, P.; Yang, H.; Zhang, L. Analysis and design of delta operator systems with nested actuator saturation. Int. J. Syst. Sci. 2015, 47, 1-7, [CrossRef]

20. Middleton, R.H.; Goodwin, G.C. Digital Control and Estimation: A Unified Approach; Prentice Hall: Upper Saddle River, NJ, USA, 1990.

21. Wang, Y.; Cao, Y.; Sun, Y. Anti-windup compensator gain design for time-delay systems with constraints. Acta Autom. Sin. 2006, 32, 455-470.

22. Zhang, J.; Peng, C.; Du, D.; Zheng, M. Improved Stability and Stabilization Criteria for Uncertain T-S Fuzzy Systems with Interval Time-Varying Delay via Discrete Wirtinger-Based Inequality. Int. J. Fuzzy Syst. 2015, 18, 784-791. [CrossRef]

23. Feuer, A.; Goodwin, G.C. Sampling in Digital Signal Processing and Control; Birkhauser: Boston, MA, USA, 1996. 
24. Goodwin, G.C.; Middleton, R.H. High speed digital signal processing and control. Proc. IEEE 1992, 80, 240-259. [CrossRef]

25. Fridman, E.; Shaked, U. Input-output approach to stability and $L_{2}$-gain analysis of systems with time-varying delays. Syst. Control Lett. 2007, 55, 1041-1053. [CrossRef]

26. Kao, C.Y.; Lincoln, B. Simple stability criteria for systems with time-varying delay. Automatica 2004, 40, 1429-1434, . [CrossRef]

27. Gu, K.; Xu, S. Small gain problem in coupled differential-difference equations, time-varying delay, and direct lyapunov method. Int. J. Robust Nonlinear Control 2011, 21, 429-451. [CrossRef]

28. El Haoussi, F.; Tissir, E.H.; Tadeo, F. Advances in the robust stabilization of neutral systems with saturating actuator. Int. J. Ecol. Dev. 2014, 28, 59-62.

29. El Fezazi, N.; El Haoussi, F.; Tissir, E.H.; Bender, F.B.; Alvarez, T. Multiclass AQMon a TCP/IP router: A control theory approach, Int. J. Nonlinear Control 2019, 1-15. [CrossRef]

30. Yang, H.; Li, X.; Liu, Z.; Zhao, L. Robust fuzzy-scheduling control for nonlinear systems subject to actuator saturation via delta operator approach. Inf. Sci. 2014, 272, 158-172, . [CrossRef]

31. Yang, H.; Xia, Y.; Geng, Q. Analyse and Synthesis of Delta Operator Systems with Actuator Saturation. In Studies in Systems, Decision and Control; Springer: Berlin/Heidelberg, Germany, 2019; Volume 193.

(C) 2019 by the authors. Licensee MDPI, Basel, Switzerland. This article is an open access article distributed under the terms and conditions of the Creative Commons Attribution (CC BY) license (http:/ / creativecommons.org/licenses/by/4.0/). 